\title{
The Perception and Cognition of Time in Balinese Music
}

\author{
ANDREW CLAY MCGRAW[1] \\ University of Richmond
}

\begin{abstract}
This article describes experiments designed to determine the perception and cognition of time-in-music among Balinese gamelan musicians. Three topics are discussed. First, the proposed connections between cultural/religious concepts of time and the construction of (time in) gamelan music are explored. Here a novel and experimental use of the Implicit Association Test is incorporated to explore potential implicit (unconscious) connections between concepts of time-in-music and time-ingeneral. Only weak associations are found. Second, the author explores music's influence in subjects' ability to gauge objective durations. Other than a tendency for Balinese subjects to consistently underestimate actual timings, and the potential for tempo changes to influence response patterns in specific ways, few strong patterns are found. Third, previously proposed models for the performance of tempo changes (here, rallentandos) are explored in the context of gamelan music. Results from perceptual tasks and an analysis of performance suggests that previous idealized models are too simplistic to describe the Balinese case.
\end{abstract}

Submitted 2007 December 18; accepted 2007 February 2.

KEYWORDS: Bali, gamelan, time, perception, cognition

THE conflation, common in musicology and anthropology, of time-in-music with time-in-general (or, time in cosmological senses) invites all sorts of well-documented problems and paradoxes (see Rowell, 1979, 1985; Mayr, 1985). Given too much time to ponder it, we are easily over-stimulated by certain eccentric truths concerning time: Definitions of time are impossible as nothing resembles it. Our concept of time is an intellectual and cultural achievement (Gibson, 1975). Pitch, timbre, and harmony are but a complex spectrum of oscillations (tempos). Music, as we know it, exists somewhere in the middle of the spectrum between the rhythmic oscillations of galaxies and those of the atom. From the physicist's perspective, music is but the play of time. As we have no organ with which to sense time directly, humans can only perceive time through the motion-reckoning of various 'clocks' (literal clocks, biological clocks, atomic decay, etc.) all of which create sound, however minute and inaudible.[2] Sound is literally time's measure (Clynes \& Walker, 1986).

Such interesting arcana thrusts us into a swirl in which the potential philosophical connections between music, time, and practically all other phenomena threatens to overwhelm; we are in danger of "losing ourselves in the infinite" (Sachs, 1953, 18). This should be checked, however, by an understanding of human modes of perception and cognition; we might conceive of time and tempo as a boundless continuum, undulating in beats from galaxy to atom, but this is not how we perceive it - such a suggestion has no cognitive reality for our sensory system. We do not perceive pitch as rhythm. The fundamental limits of our short- and long-term memories have profound implications for how we understand and experience the difference between the groove,[3] rhythm, meter, form and overall structure of music. In this article I work to tease apart the conceived and perceived natures of temporality[4] in Balinese music.[5]

This article revolves around a few questions: What does it mean to describe Balinese music as cyclical? Is Balinese time-in-music iconic of Balinese worldviews about time-in-general, as has been previously suggested? For the Balinese, does time-in-music distort other, more ostensibly subjective, experiences of time? If so, in what ways? Do temporal transformations in Balinese music follow previously proposed models which suggest a connection between kinetic and musical imaging in the mind? I approach these questions through three lines of inquiry: 1) cognitive/(ethno)musicological, 2) perceptual/empirical and 3) theoretical/perceptual. First, I explore the question of cyclicity and the possible iconicity of Balinese time-in-music with regards to Balinese worldview. Here I incorporate a contemporary method of cognitive testing, the Implicit Association Test. Second, I explore the interaction between musical and mundane time perception of Balinese music through a test involving duration. Finally, I investigate the 
construction of temporal changes (specifically, rallentandos) in Balinese music. This section is primarily theoretical and perceptual and deals with previous theories which have suggested a biological, and so crosscultural, theory of tempo transformations and relations.

\section{CROSS-DOMAIN MAPPING AND THE TIME-IN-MUSIC, TIME-IN-GENERAL ASSOCIATION}

(Ethno)musicologists including Amatzia (2001, 2006), Becker (1981), and Seeger (1980), among others, have suggested that a culture's worldviews of time, space, nature, and society are sometimes mapped onto the domain of music. 'Cross-domain mapping,' the more common term for 'intra-modal structural analogies' - thinking of one set of phenomena in terms of another-has been considered in music theory and cognitive musicology and ethnomusicology since the 1980s.[6] Cross-domain mapping, which is encountered in both music itself (i.e., music painting) and speech about music (i.e., tonal center as exerting gravitational force) is a kind of metaphor often based in image schemata and typically grounded in bodily experience.[7] In both Western and Balinese traditions, music and especially time-in-music are associated with concepts of physical motion and position. Western definitions of time and music in terms of motion are ancient [8] and the tendency for the mind to activate visual and kinetic imagery through auditory stimuli seems deeply embedded, possibly universally hard-wired. Analytical work focused on the function of kinetic cross-domain mapping in perception, rather than in performance, suggests that the experience of music often occurs as metaphoric motion, what Clarke (2001) calls "fictional movements" in the virtual space of the mind. However, while our brains may be hard-wired to allow for the strong interaction of auditory and kinetic/visual processing, the exact ways in which this happens may be more culturally determined.

Gravity (towards tonal center, for instance) has been a favored metaphor in Western music theory[9] while concepts of wave and flow pervade Balinese folk and academic conceptions. The Balinese term for wave, ombak, is interesting in this context in that it represents what may be a rare example of non-human motion mapping in music theory.[10] In Bahasa Bali (Balinese) and Indonesian the term ombak refers primarily to the imagery of the ocean's wave but in musical contexts refers to qualities of temporality, dynamics, melody, and intonation. The architectural, organic, and linguistic models that pervade the textbased tradition of Western music theory serve the analysis of music as a complete structure, as in notation, but terms such as ombak (and groove), as an expression of primarily oral traditions, more neatly fit the experience of and discourse about music as it arrives.

The identification of homologies between music structure and cultural worldview by ethnomusicologists and cultural anthropologists is similar to cross-domain-mapping, but on a vastly larger and more complex level. We might refer to cross-domain mapping as the cognition of limited information from one-perceptual apparatus in terms of another as in the conception of decreasing musical tempo (auditory) as a sprinter's slowing gate (kinetic/visual) or of the destructive interference caused by Balinese paired tuning (auditory) as the flow of waves in water (visual). The connection of music to worldview involves the inter-domain transfer of larger schema, or collections of schema. Cultural anthropologists and ethnomusicologists, including those mentioned above, have suggested that such schema add semantic coherence to life and imbue otherwise disconnected phenomena with greater meaning.

\section{The Iconic 'Cyclicity’ of Balinese Music?}

Several authors have suggested that the temporal organization of time-in-music is directly and deeply (implicitly) connected to the conceived nature of time-in-general in specific cultures, elevating it even to the level of mythic significance (see, for instance, Maceda, 1986; Rowell, 1979). It is seductive to think of Balinese music in terms that accord with assumed Balinese worldview and concepts of time-in-general just as many scholars have been tempted to connect Indian concepts of tal rhythmic forms with the Hindu cosmological notion of kalpa, or world cycles.

In Judith Becker's short but powerful article 'Hindu-Buddhist time in Javanese music' (1981), structural form in certain kinds of older gamelan musics[11] are described as being iconic of calendrical and cosmological cycles in Balinese and Javanese culture. According to Becker, gamelan music reflects older, pre-Islamic notions of worldview: "the music of the gamelan is deeply imbued with Hindu-Buddhist conceptions of reality, and foremost among these is the concept of time" $(1981,163)$. Becker suggests that time in gamelan music is non-teleological (here tonal harmony serves as a teleological foil in contrast to gamelan music), and is "not dependent upon the perception of change, but rather its opposite, no change, 
the intimation of eternity or Nirvana, the sense of being outside of any time framework at all" (ibid). Becker dates her example to pre-Islamic, Hindu-Buddhist Java (pre-fourteenth century)[12] suggesting that while "many modern Javanese no longer subscribe to the [non-teleological] epistemology in which it was conceived," its continued performance may still "evoke a response in modern Javanese listeners and musicians" (p.164). However, upon its arrival, Islam, its worldviews and notions of time (teleological, based on an ultimate judgment day) did not replace Javanese notions, but blended complexly with them, just as the Ramayana has coexisted alongside the Al-Quran for centuries in Java. Further complicating the assertion that religious worldview dictates musical structure is in this case is the fact that musical textures that can be described as teleological and non-teleological have existed in gamelan music on both islands before and after Western (Islamic and European/colonial) contact.

Balinese music is cyclic, or non-teleological, non-processive, only in the way that time itself is; it can seem to repeat but does not exhibit literal repetition in the way motion or physical structure can. Timein-music is never so literally cyclical as are physical representations of time-in-general, such as a calendar or clock. Clayton points out that the concept of cyclicity in music is a metaphor "used in order to clarify, mediate, and communicate subjective musical experience. Musical time is no more a circle, or a wheel. . . than it is ... a wave" (Clayton, 2000, 19). Balinese gong patterns, for instance, seem conceptually cyclical because any instance shares structural, architectural features with elements of subsequent music; although the experience of the two instances cannot be literally the same. In classical Indian cosmology the kalpa (cosmic ages) repeat, but not in detail, just as do Indian tala rhythmic forms and Balinese gong patterns.

In his discussion of the frequent characterization of tal as connected to deeply embedded Hindu concepts of cosmological time Clayton (2000) outlines the problems of perception and predisposition:

... if music reflects deep cultural patterns in any meaningful way then those patterns must be perceived by the listener, and yet a listener may perceive such patterns partly because of a predisposition to interpret any event in terms of a limited number of archetypal patterns ... moreover he may be inclined to hear certain things in music because he has learned from the prevailing ideology that they are expected to be there. . . (2000, p.7).

For Clayton, music cannot simply reflect time-in-general and ideology cannot be determinant of musical structure because music is such a complex social phenomena that simple reflectionism is untenable (2000, p.22). Middleton (1990) notes that such structural homologies are problematic because they are often circular (for they explain each other), lack historicity, and assume a whole, integrated and functional culture. In Bali, cultural ideologies concerning temporality have certainly changed throughout history, even radically over the past century. If time-in-music was iconic of cultural worldviews about time-ingeneral then we might expect more change in musical style than has occurred over the past century. But slow ceremonial lelambatan are performed today much as they were before the massive effects of colonialism and globalization were felt.[13]

However, there nevertheless may be connections between musical and mundane temporalities at the level of the unconscious, rather than in the conscious mind, where its presence and function would be hard to detect and measure. While we may provisionally discount worldview as determinative of the construction of time-in-music in Bali, we can wonder to what extent elements of musical structure might be iconic of more general ideas in the mind of the musician. Are time-in-music and time-in-general implicitly (unconsciously) associated in the minds of Balinese musicians? Is time-in-music, as Epstein (1995) suggested, a special case of time-in-general, and therefore processed in the mind in the same manner as all durational information?

\section{Implicit Associations}

Mind is a donkey, words are a horse. . . Mind and words arriving 'part-way' is being-

time. . .

(Dogen Zenji, 1200-1253)

Musical expression is a repository of culturally specific ideas which come to us subliminally, thus unselected, unfiltered, and uncensored. Herein lies its power. (Judith Becker, 1981). 
In the mid-nineteenth century, F.C. Donders discovered that the "time required to perform a mental computation reveals something fundamental about how the mind works" (Greenwald et al., 2007, 59). That is, latency in response is a sign of implicit (unconscious) attitudes; "the easier the mental task, the quicker the decision point is reached and the fewer errors that result" (ibid). For decades, psychologists have used response time as a variable to understand the nature of cognition in dozens of experimental methods.[14] The Implicit Association Test (IAT), developed over the past decade by cognitive researchers, similarly measures response time to estimate the strength of associations between concepts and attributes to determine an individual difference measure of implicit social cognition. The IAT obtains response times by measuring the time-lag between the appearance of an attribute in the middle of a display screen and the subject's pressing a key on a computer keyboard to assign the attribute to one of two possible categories.[15]

In constructing an IAT test to investigate the connections between time-in-general and time-inmusic, we cannot simply ask if Balinese time-in-music is iconic of time-in-general. Instead we must ask if time-in-music is more strongly associated with time-in-general than it is with some other category such as dance or space. An IAT test was modified and administered to several Balinese musicians. Subjects were asked to arrange the terms: year, month, week, tumpek, minute, hour, front, above, right, left, behind and below into the categories: 'time' or 'position.' Next, the subjects were asked to arrange the terms: agem, seledet, baris, malpal, miles, tanjek, gongan, gineman, tabuh telu, tempo, suwud and bapang into the categories 'dance' or 'music'.[16] In Cross-Alignment Task 1, subjects are then asked to align the above terms into one of two columns: A) music or position, or B) time or dance. In Cross-Alignment Task 2, subjects are asked to align the above terms into switched columns: A) time (waktu) or music (music), or B) position (spasi, posisi) or dance (tari).[17] Categories 'music' and 'dance' are never aligned in this test.

TEKAN 'd' UNTUK

Waktu

ATAU

Musik
TEKAN ' $k$ ' UNTUK

\section{Spasi / Posisi}

ATAU

Tari

\section{tabuh telu}

Figure 1. Screenshot from the Indonesian IAT test: Cross-Alignment Task 2. 'Press "d" for Time or Music, Press "k" for Position or Dance.' 


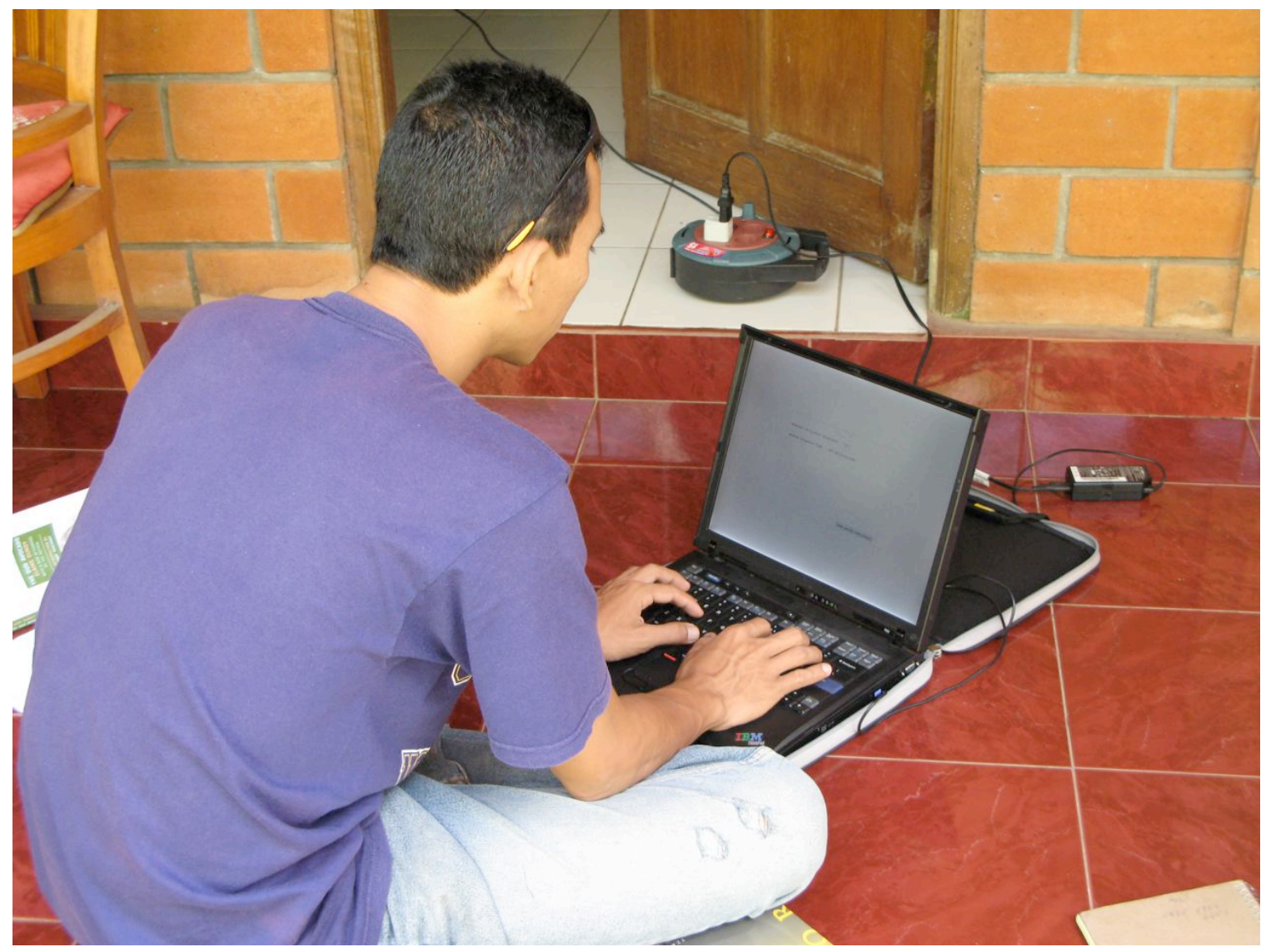

Figure 2. The Balinese composer and performer Ida Bagus Widnyana takes the modified IAT test in Peliatan, Bali, Indonesia, August 2007.

Faster response in associating music and time terms in Task 2 would signal an implicit cognitive link between concepts of time and music. Little or no latency difference between Cross Alignment Tasks 1 and 2 would suggest that concepts of time and music (and dance and space) are no more strongly associated than, say, concepts of time with dance or music with space. To further test the cognitive reality of the claimed cross-domain iconicity of Balinese time and music, a second test was designed in which the category 'dance' was replaced with a more neutral category of 'instruments' in case associations between dance and space were potentially stronger, and thus masking, associations between music and time. Categories 'music' and 'instruments' are never aligned in this test.

Based on preliminary testing of a limited number of Balinese musicians,[18] the tests displayed a mild negative correlation between time and music and time in general (or a slightly stronger preference for associations between dance and time-in-general) in the first test and a slight correlation between time-inmusic and time-in-general on the second test.[19] Significantly, the tests displayed a rather wide variety in response times and tendencies between subjects suggesting that there is far from a homogenous cognitive mapping among native performers as a group. In general, time-in-music may be regarded as weakly implicitly iconic of time-in-general among Balinese musicians; however musicians themselves differ widely in their responses. The use of the IAT in this context is novel and the author is not a psychologist; the information here should be understood as preliminary and is meant to suggest future avenues for research. The use of the IAT test in this context raises several potentially problematic issues, among them being that the IAT test is based upon the rapid cognition of words, not of music itself and it does not necessarily follow that we can extend the IAT results to experiences of time and music.[20]

The associations reported in IAT tests often differ from subjects' self-reported, explicit attitudes and beliefs. While formal, questionnaire-based verbal reporting was not an aspect of the test described here, the author held informal interviews with each of the subjects on the topic of the iconicity of time in music. All agreed with the veracity of Becker's notion of the iconicity of musical and general time, however the IAT suggested only weak iconicity. Interestingly, while traditional views of the implicit system suggest that it is slow to learn associations and consequently slow to change, recent research 
suggests that IAT measures shift in response to situational cues. That is, implicit attitudes are not completely stable characteristics; they sometimes vary in relation to current situation and new learning.[21] In retrospect, it is possible that the informal conversations with informants on the topic prior to the test influenced their responses.[22] Such variability flies in the face of common assumptions about unconscious associations, beliefs, or attitudes as being more 'true' or 'real' than explicit ones. Indeed, participants are often surprised by their IAT scores, suggesting that "the IAT taps attitudes or beliefs that are not accessible by conscious introspection" (ibid). Explicit and implicit cognitions exist simultaneously, neither 'truer' than the other; the more interesting investigation concerns the complex relationship between the two and how they interact to determine behavior and performance.[23]

\section{PERCEPTION OF DURATION}

A central model of the perception of time-in-music suggests that perceived duration depends on the amount of information stored or processed (Fraisse, 1963; Ornstein, 1969; Michon, 1972). Simply stated, denser textures are often perceived as comparatively longer in duration.[24] Information processing relates more directly to the perception of meter and form (durations longer than 'surface' level rhythms) than to the perception of tempo. Investigations of memory's role in the perception and cognition of music have impacted theories of meter and the understanding of tonal hierarchy (London, 2002, 529). Meter is often described as a periodic hierarchy of pulsation existing within the bounds of the perceptual present- shortterm memory lying between $100 \mathrm{~ms}$ to $6 \mathrm{~s}$ (ibid) — while elements of form exist within stored and retrieved long-term memory. Long-term memory stores the event hierarchies of several works and eventually, through cognition, gives rise to a sense and understanding of stylistic structures (such as Western tonal harmony or Balinese extended gong form).

The music theorist and composer Jonathan Kramer stated that he was less interested in the time a piece takes than in the time it 'invokes' $(1988,7)$. Perceptual and music-theoretical studies have suggested that music creates, or exists, in special kinds of time and that it can distort 'objective' (rational, clockbased) perceptions of time. Empirical experiments typically concern the perception of miniscule bits of synthesized sounds, not necessarily music as experienced in culture. Ethnomusicologists warn against the kinds of artifacts that ecologically invalid perceptual experiments might produce, suggesting that actual music is experienced in social, cultural contexts that provide non-musical indications of the flow of time (e.g., conversation, noise, your watch, your next appointment etc.). These two kinds of time, musical and mundane, can be conceived as existing in a kind of stereo temporal image in the mind. Like stereo audio, we can actively shift our attention to focus upon one temporal stimulus or another.

A simple test was administered to gauge Balinese musicians' abilities to reckon objective durations while listening to gamelan music in social contexts. Subjects listened to ten short examples of gamelan performances and were asked to indicate on a time-line how long they felt the example lasted. Odd-numbered subjects were asked to perform the task in reverse to offset any effects of fatigue, timeorder errors, or influence from previous audio examples.[25] This experiment gauged so called prospective timing among musicians. That is, the musicians knew in advance that they were going to be asked to gauge the length of the examples and so likely attended to objective time more often and more deeply than listeners would in normal listening experiences. Prospective timing (also called 'experienced duration') experiments are contrasted with retrospective timing experiments in which listeners are only asked to estimate timings after the fact. Based on a previous meta-analytical study (see Block and Zakay, 1997), it seems that prospective and retrospective timings may involve different cognitive processes (ibid, 185). The prospective paradigm seems more easily influenced by amounts of information within stimuli than retrospective timings; prospective timings are also more accurate.

The perception of duration in Balinese music is intricately connected to rhythmic, structural, melodic, and orchestral qualities. Michael Tenzer (2000) employs the Balinese terms majalan, a verb meaning to move forward (from jalan, to walk) and ngubeng an adjective suggesting stasis or to "spin one's wheels" (Tenzer, 2000, 178) to describe melodic quality. In the test described here, subjects often rated textures that could be described as majalan (changing) as longer than ngubeng (static, repetitive) textures in examples of comparable length. For instance, example nine shown in Table 1 below includes an extended, complex and ametrical, majalan kebyar introduction from the classic work Teruna Jaya; example ten consists of a less dense, more staid, ngubeng section of lelambatan Tabuh Pat Jagul. 33\% of subjects overestimated the duration of example nine (with a standard deviation of $+22 \%$ ) while only $5 \%$ of subjects overestimated the duration of example ten (with a standard deviation of $+20 \%$ ). This finding is in line Kramer's research which suggested that perception of duration increases with increased information (Kramer, 1988, 337). However, while all of Kramer's subjects “consistently represented [denser examples] 
as about $15 \%$ longer" than actual, this is hardly the case among Balinese subjects. While more subjects rated the denser example nine as longer than example 10, an overwhelming majority of subjects underestimated the duration of both examples nine $(67 \%)$ and ten $(86 \%)$. In fact, in all but one example the majority of subjects underestimated actual durations.

Besides suggesting that these subjects really like gamelan, so much so that merely listening to it makes time fly by even faster than usual, it is unclear what such a result could mean. However, in a limited number of tests on American musicians, unfamiliar with gamelan music, a nearly equal majority overestimated the same examples, suggesting that their difficulty in parsing an unfamiliar musical language expanded their sense of overall duration. That Americans would consistently overestimate gamelan timings and that more Balinese tend to overestimate the more complex example nine (Taruna Jaya) over the less complex, and equally long example ten (Tabuh Pat Jagul) seems to conflict with the findings of Block and Zakay (1997) whose meta-analytic study of twenty experiments found that as task difficulty increased, experienced duration decreases. Block and Zakay suggest that "as processing difficulty increases, opportunities to attend to time decrease" $(1997,188)$.

Only in example seven, which includes an extended accelerando, did the majority of subjects overestimate the duration.[26] This suggests that changing tempos, besides information, can influence perceived duration. Furthermore, in steady examples the comparative strength of deviation from actual timings decreased as the examples themselves increased in length. In other words, subjects were more accurate the longer the example was, despite changes in density or tempo, which seems counterintuitive.

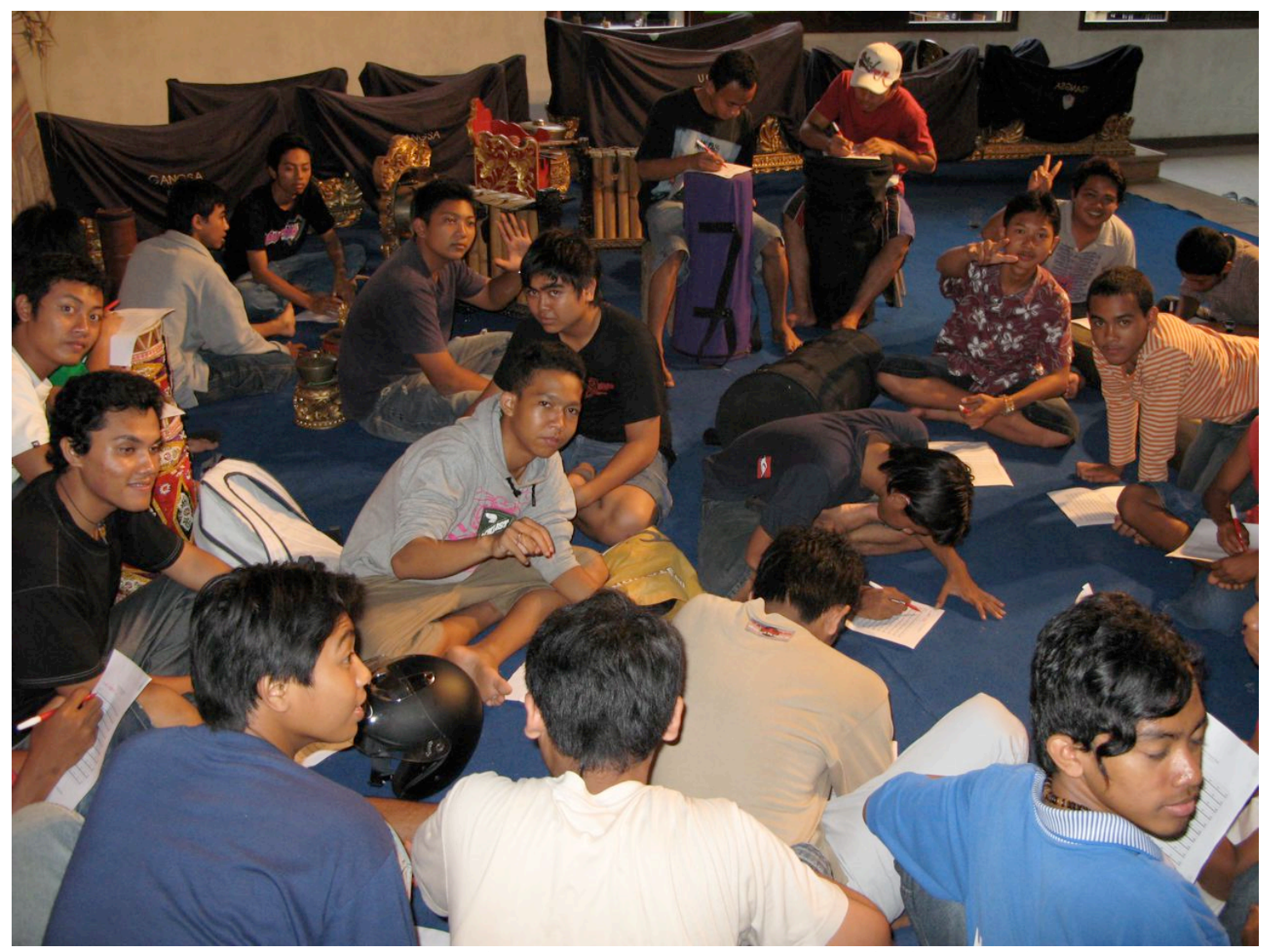

Figure 3. Young performers from gamelan Chandra Wirabhuana from Ubud, Bali, Indonesia take the duration perception tests, August 2007. 
Table 1. Perceived duration among Balinese musicians of ten short musical examples.

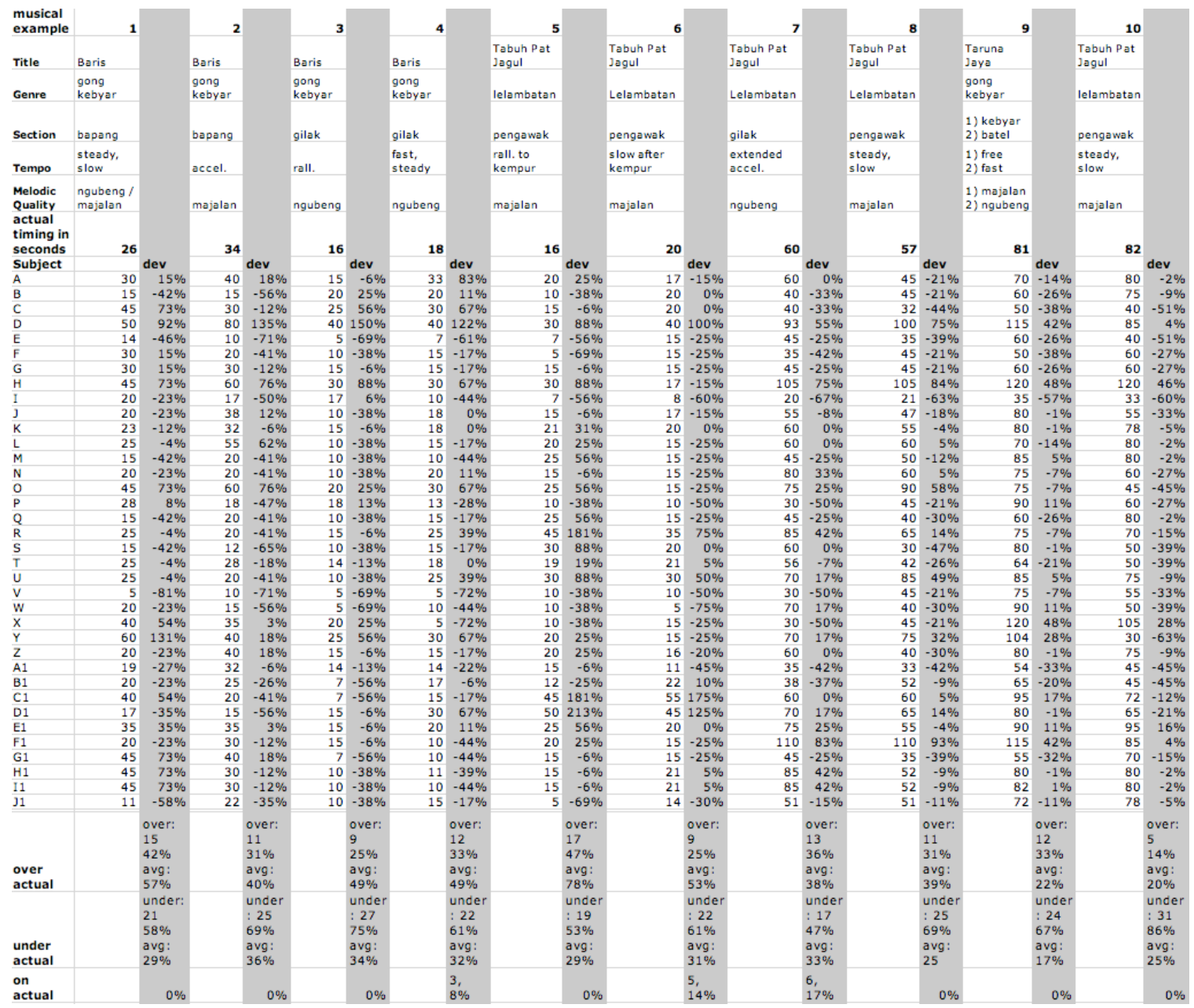


Table 2. Comparison of perceived duration by musical qualities.

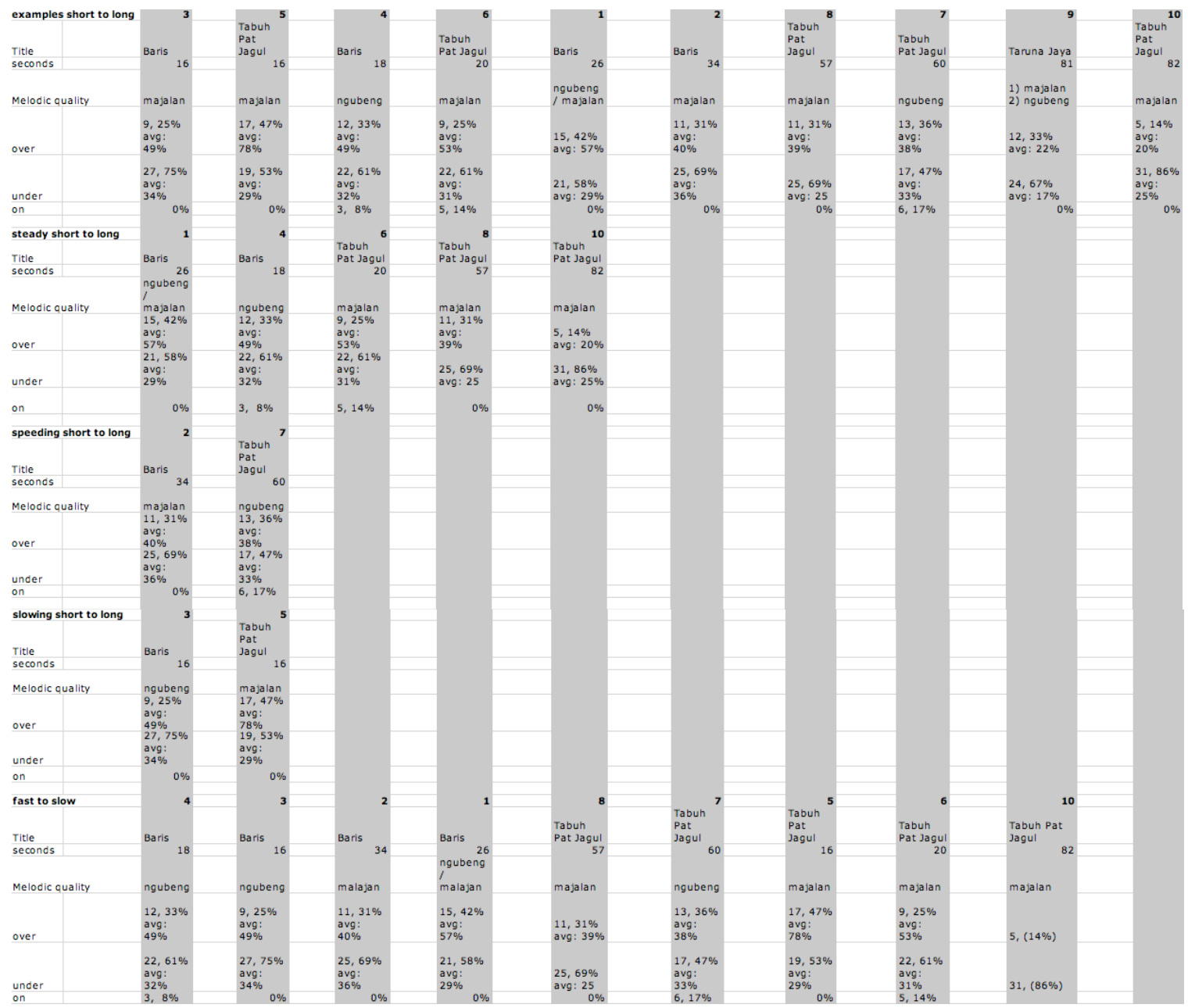

TEMPO TRANSFORMATIONS (RALLENTANDO) IN BALINESE MUSIC

To what extent might the structure of tempos and tempo changes in Balinese music be biologically based and therefore exhibit certain culturally universal tendencies or abilities? David Epstein strongly suggested that proportional tempos[27] and certain specific shapes of accelerandos and rallentandos are universal and rooted in human biology. Epstein attempted to escape "individual modes of perception" in musical analysis by connecting musical performance and perception to biological, rather than cognitive, functions; "If there is a biological basis for a theory. . . the discussion moves to a higher plane of certainty, relevance and significance" (1985, 38-39). According to Epstein, as in Chomskian models of language ability, we have a common innate, universal competence regarding the structure and regulation of tempo in music; overlain this innate ability are varying levels of talent and ability. This model downplays cultural (topdown) modes of perception and suggests that models that diverge from suggested biologically based universals are either 'unnatural' or are due to poor performance.[28]

The suggestion that biological clocks, such as the heartbeat, function as pacemakers in musical contexts has long been discredited on the grounds that such biorhythms are variable and influenced by external stimuli (Sachs, 1953; Kramer, 1988). This notion has been replaced by complex, variable, taskrelated clock-models. If we do have clocks embedded somewhere in our central nervous system or subconscious (Clynes \& Walker, 1986), they are flexible, context sensitive (Povel \& Essens, 1985) and can be influenced by musical training and culture (Rammsayer \& Altenmuller, 2006). Musicians must have some kind of internal clock if some of us can accurately gauge tempo, but this kind of reckoning, which is not embedded or unitary, seems related only to learned, skilled motor tasks, or imagined motor-tasks (Treisman, 1964; Kristofferson, 1980). 
Feldman et al. (1992) propose that in 'expert' performances of music (Western and non-Western) tempo changes follow not linear nor exponential models, as has been previously suggested, (Sundberg \& Verrillo, 1980), but a model in which the progression and change of tempo over time is "controlled by a mental analog of mechanical force" (Feldman et al., 1992, 185). The authors found that a more "consummate aesthetic proportion" of tempo change followed a force model that was either linear (creating a quadratic curve) or parabolic (creating a cubic spline curve). In this model music moves along at a constant pace unless impelled to do otherwise by a new force. That is, tempo is controlled not by biorhythms, but as a result of a cross-domain mapping between kinetic and musical cognitive processes at the level of the subconscious. The authors suggest that: ". . . the matches found here between observed profiles and theoretical ideal shapes suggest that performers actually conform in detail to mathematical constraints of which they presumable have no conscious knowledge. If this conformance actually were presumably responsible for the aesthetically satisfying paradigm for 'beauty,' or at least naturalness of form, that is refreshingly concrete" (p.203).

In a simple experiment Balinese musicians were asked to rate three different kinds of tempo change. Subjects listened to a recording of the traditional piece Bapang Selisir transcribed in Finale outputting samples of Balinese gamelan. Three versions of the work were performed in which a rallentando conformed to either a linear, quadratic, or cubic spline (force-model) curve.[29] Subjects were asked to rank their preference or to indicate if they felt that: A) the examples were musically equivalent, B) that there was no perceivable difference between them, or C) that none felt musical. Odd numbered subjects heard the examples in reverse order.

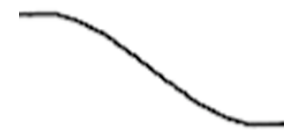

Figure 1. Cubic spline curve.

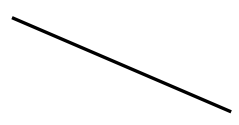

Figure 2. Linear curve.

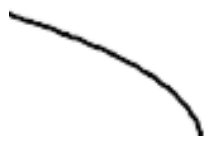

Figure 3. Quadratic curve.

Table 3. Perception and preference of tempo changes, rallentandos.

\begin{tabular}{|c|c|c|}
\hline \multicolumn{2}{|c|}{ Perception of Rall. Qualities } & \multirow[b]{2}{*}{$31 \%$} \\
\hline Spline & 11 & \\
\hline Quadratic & 5 & $14 \%$ \\
\hline Linear & 12 & $33 \%$ \\
\hline All Viable & 2 & $6 \%$ \\
\hline No Difference & 2 & $6 \%$ \\
\hline None Viable & 4 & $11 \%$ \\
\hline
\end{tabular}

Table 4. Actual, performed tempo changes (rallentandos) in ten traditional Balinese gamelan works.[30]

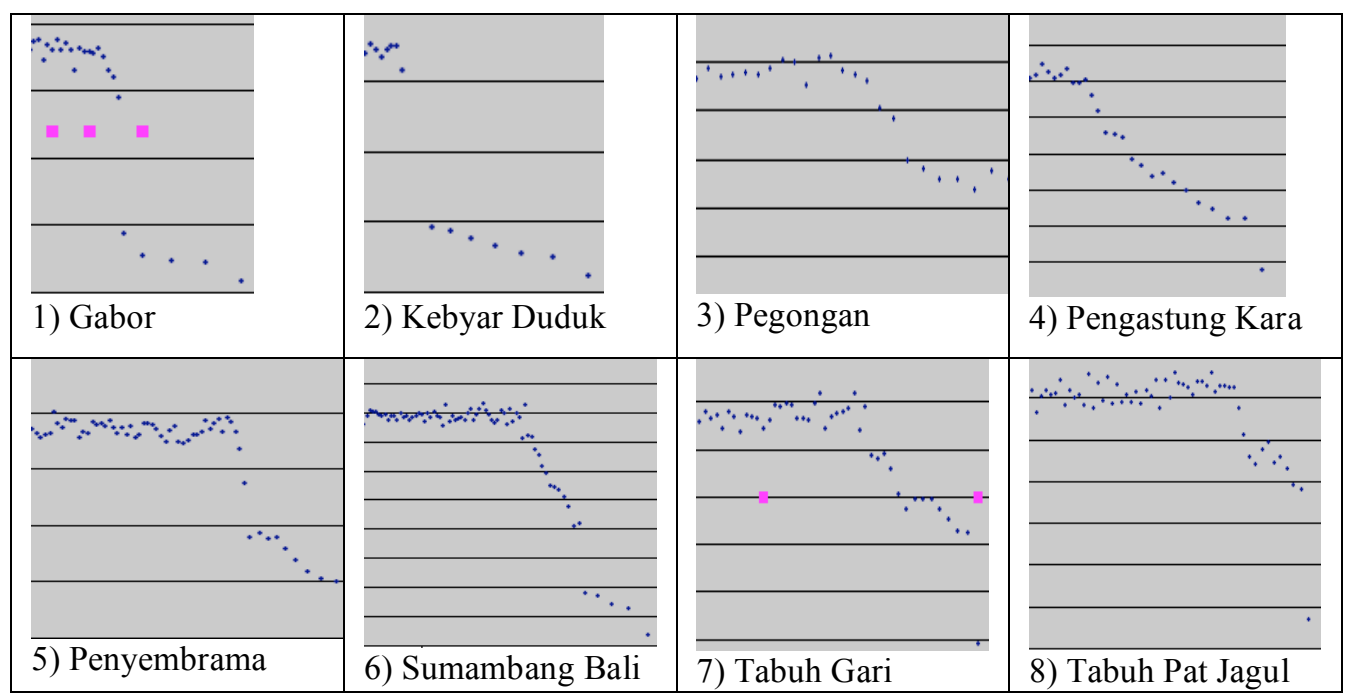




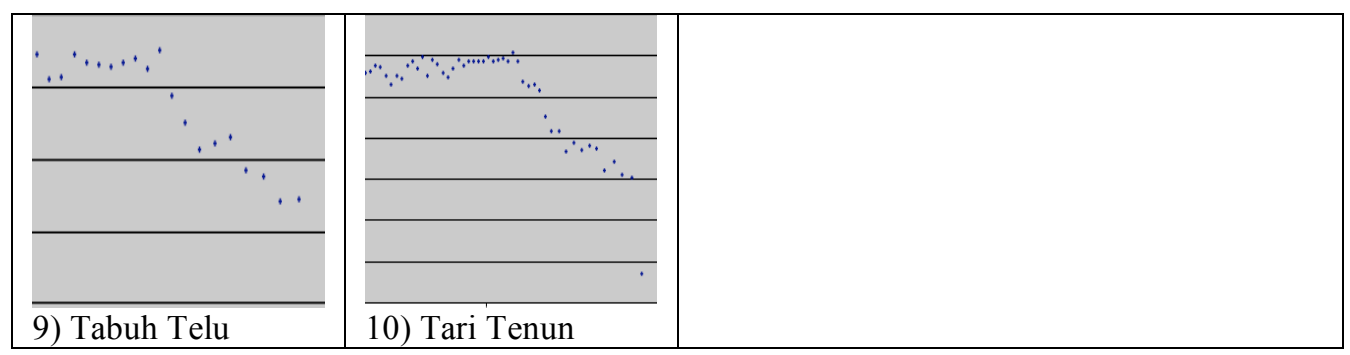

As seen in Table 3 a majority of the subjects chose linear curves, that model suggested by Epstein (and others) to be the least 'natural.' Nearly the same number chose the cubic spline example, and only a few chose the, supposedly intermediate, quadratic example. Several examples of ending rallentandos from actual, performed music are included in the examples in Table 4. While none approach the simplicity and neatness of the idealized curves presented in Figures 1-3, several similarities can be seen; Gabor closely models a cubic spline curve, while Kebyar Duduk seems linear. However, similarities to idealized models are only found in sections of what are actually multi-part temporal transformations. In fact, each rallentando has several stages; a sudden drop in tempo followed by a temporary stabilization (if not momentary increase, as clearly seen in Penyembrama, Tabuh Gari and Tabuh Telu) followed by a curve resembling the second half of either a cubic spline (Penyembrama, Tabuh Gari) or quadratic curve (Pengastung Kara, Sumambang Bali). Balinese rallentandos do not follow simple paths but combine elements of several different idealized models; like Balinese rice fields, the tempo transformations in these examples are terraced.

\section{CONCLUSION}

The linking of musical and cosmological, or mundane, time seems to be a desideratum to the postenlightenment mind. Inter-domain cultural schemas (icons) are attractive because they are "simplified world(s) that abstract from the manifold complexities of the real world" and "bring different domains of experience into alignment" (Perlman, 2004, 16). The linking of music with extramusical concepts and images (of time, mandala or calendars, for instance) serves as an incredibly powerful mnemonic and pedagogical tool, however difficult it might be to prove such connections as directly causal or reflective. Such homologies can be argued against from various anthropological and historical viewpoints, but the most interesting evidence may come from cognitive investigations. Here, cognitive experiments suggest that evidence for the unity of time-in-music and time-in-general in the mind is weak and may be balanced by other, equally strong associations.

In the simple tests concerning perceived duration described here few patterns emerge from the data. A more accurate result regarding the influence of tempo changes on perceived duration might be produced from a test that was limited to only examples that included a single type of tempo change, in a single musical context. Further tests might explore the role of anticipation; such as influence of majalan textures leading to important structural moments such as the end of gong patterns.[31] However, we should be wary of tailoring potentially ecologically invalid experiments simply to produce rational response patterns. The very vagueness of this data may demonstrate a simple but powerful point; the perception of music, and especially of time-in-music, is extremely complex, fluid, and individualized. A journal of null results in cognitive and empirical musicology might be just as valuable as a catalog of intriguing patterns.

Rather than biological, information-based (cognitive). and cultural models competing, the perception of time-in-music is more likely the result of "convergent information from different sources, with one or the other source dominating in different conditions" (Clarke \& Krumhans1, 1990, 220). The consensus emerging from recent empirical studies suggests that the perception of time-in-music is extremely complex and arises from a variety of sources and processes. These modes of perception are partially learned (i.e., top-down, especially so for musicians) and can be adjusted through the active manipulation of the subjects' concentration. The analysis above of tempo relations in Balinese music demonstrate the culturally learned, and more unpredictable, modes of both perceiving and performing timein-music. 


\section{NOTES}

[1] School of Arts and Sciences, University of Richmond, USA.

[2] According to Bharata, the putative author of the fifth century Indian Natyasastra: "There is no sound which is outside time's measure and no time measure without sound" (quoted in Clayton, 2000, 13).

[3] I define groove as the subnotational negotiations of rhythm and timing in multi-instrumental contexts. Concepts of groove have been seriously studied in by scholars including Keil (1995) and Zagorski-Thomas (2007) although it has never been seriously investigated in studies of Balinese music.

[4] I use the term 'temporality' to indicate all elements of time, duration, and rate in music. Temporality includes the overall duration of a piece, its tempos and their transformations, constructions of meter, density and the various ways in which a piece of music can distort the 'objective' perception of time through the manipulation of information, i.e., repetition and variation versus the presentation of new material.

[5] Since Colin McPhee's opus on Balinese music was published in 1966 until the present 'Balinese music' has generally been synonymous with traditional gamelan percussion orchestra music. Today the ecology of the Balinese musical landscape is considerably richer than during McPhee's era; Balinese rock, punk, jazz, experimental, and pop bands exist alongside and combine with gamelan. In this article, I am referring specifically to the construction of temporality in traditional kebyar, gong gede, semar pegulingan, and pelegongan gamelan repertoires.

[6] The obvious example in music is thinking of pitch in terms of height in Western musical cultures: the faster the oscillation, the higher in a vertical axis the pitch is imagined to reside. The Balinese context helps to illustrate how cross-domain mappings are culturally based; the Balinese imagine pitch in terms of sizes, the faster the oscillation the 'smaller' the pitch is imagined to be.

[7] Image schema are employed by Feld (1981) in reference to the Kaluli, by Zemp (1979) in reference to the Are'are, and by Walser (1991) in his discussion of distortion. Mark Johnson's (1987, 2003) philosophical discussion of the bodily basis of cognition deals with this topic in great depth. See also Perlman (2004).

[8] Aristotle defined time as the measure of motion, the mensura motus. The most obvious cross-domain mapping here would be the tendency to connect musical tempo and speed of human motion; this connection is so basic that it is difficult to even think of it as a cross-domain mapping.

[9] Thomas Christensen discusses the early use of Newtonian theories of gravity to describe aspects of tonality in the theories of Jean-Philippe Rameau (1683-1764) (Christensen 1993, 109).

[10] Concepts of waves in Western music theory do exist, as in Zuckerkandl's (1956) 'wave theory' of meter. In this theory meter is conceptualized as going away and coming back along a temporal continuum. However, such 'flow' theories are rare as compared to physical, architectural concepts of temporal construction in Western music. See also Feld's description of the Kaluli concept of musical waterfalls (1982).

[11] Becker refers to the Javanese piece Langen Bronto (Branta), a ladrang in the pelog lima mode. See: http://muse.calarts.edu/ drummond/pdf/p5/LangenBranta.pdf for notation.

[12] The central Javanese towns of Yogyakarta and Surakarta (Solo) are considered the musical heart of the Central Javanese gamelan tradition. Prior to the mid-eighteenth century these two towns were ruled as a single kingdom, during which time the musical repertoire between the two locals were shared. Becker's example, Langen Bronto, exists only in Yogyakarta today, and in Solo is only known as a 'Yogyanese' work suggesting that it is a more recent (post mid-eighteenth century) addition to the Yogyanese canon. 
The first indigenous reference of the work this author is aware of (from the Solonese Wedhapradangga) cites its performance in the 1930s.

[13] However, contemporary local discourses of conservative traditionalism exert a powerful force upon musical change in Bali. As Seebass (1996) has suggested, the emergence of the twentieth century gong kebyar repertoire, with its unique construction of temporality as compared to previous Balinese repertoires, emerged at the historical rupture of sustained Western contact in Bali. Rowell suggests that music and ideology exist in a "mutual feedback system" $(1988,330)$ and that symbolic representations (i.e., music) often manifest with a time-lag "so that music may come to 'reflect' ideas which have been superseded in other realms of thought" (quoted in Clayton 2000, 17).

[14] Greenwald lists the Stroop task, episodic or repetition priming, semantic priming, evaluation priming "and many others to asses attention, perception, memory and categorization" (Greenwald et al., 2007, 60).

[15] The IAT was designed primarily Dr. Anthony Greenwald at the University of Washington. See Greenwald et al. (2007). While the test has been customized for many different experiments, the most widely-known experiments are those that suggest a common implicit association among Anglo-Americans between African Americans and criminal activity. See http://implicit.harvard.edu. The test was first published in 1998 and has since undergone improvements and modifications. As of 2007 over 200 papers report use of the method and 4.5 million tests have been taken online (Greenwald et al., 2007, 61). Most non-Web-based versions of the test run in the Inquisit program, an application designed to precisely measure response times in computer-based tests.

[16] The tests were conducted entirely in Indonesian. Tumpek is a special event in the Balinese calendrical system in which two calendrical cycles coincide (as in Friday the $13^{\text {th }}$ ). Gineman is an instrumental overture; tabuh telu is a structural form; suwud refers to endings; bapang is a structural form; gongan is a generic name for structural forms outlined by the gong. Musical terms were chosen for their 'cyclical' or 'iconic' characteristics as described in Becker and Becker (1981). Agem is a stylized dance pose; seledet is a sudden eye-movement; baris is a male dance; malpal refers to walking in dance forms; miles is a movement out of the agem position; tanjek is a foot plant moving out of a miles into a following agem pose.

[17] Subjects repeat each task switched from right to left to account for potential artifacts from lefthandedness or dyslexia.

[18] Although the sample size here is limited (12 subjects), it should be pointed out that the IAT is intended to gauge, and report, individual rather than cultural attitudes and associations.

[19] The method of reporting in the freely distributed (and modifiable) IAT test is counterintuitive; positive integers indicate a stronger association (shorter response/latency timings) between anticipated opposed categories, in this case time-in-general and instrument in test 1 and time-in-general and dance in test two; negative integers indicate a stronger association between anticipated matched categories, in this case timein-music and time-in-general in both tests 1 and 2 . The average correlation in the first test was .111, in the second, -.261 . Previous IAT tests suggests that strong associations between anticipated matched categories are generally in the -.800 range. In both tests described here, individual subjects' responses ranged from moderately positive to moderately negative responses. For more on the method of reporting and the kinds of correlations applied by the testing algorithm, see Greenwald et al. (2007).

[20] Furthermore, it is taken for granted here that such a thing as the unconscious exists and that it functions in the same way cross-culturally.

[21] "For example, race bias (as measured by the IAT) decreased after participants viewed pictures of admired African Americans and disliked White Americans, and such effects persisted after a 24-hour period) (Greenwald et al., 2007, 81).

[22] Such variability need not represent a challenge to the method's validity, however. "Just as explicit attitudes may shift in response to the current situation, a particular situation may activate a specific set of 
associations, temporarily making certain category-target associations stronger" (Greenwald et al., 2007, 82).

[23] To this author's knowledge this is the first instance of the use of the IAT test in gauging attitudes and associations relative to musical structure (however, see Rudman et al., 2002). The use of the IAT in this context is atypical and complex. Further research might focus on simpler, and more easily defined attitudes towards musical textures. Relative strength between harmonic or timbrel qualities and gender (i.e., distortion and masculinity), for example, may produce interesting results. Because the test is based upon latency, incorporating the perception and experience of actual music is highly problematic. However, a test could theoretically be developed in which timbre, the most immediately available musical parameter (allowing for very short sound files), was tested against specific attributes and attitudes.

[24] The Balinese karya gede ceremony, in which several gamelan ensembles perform simultaneously but independently in a confined space, has been mentioned by several informants to create a sense of timelessness. It seems that auditory information overflow can reach a level at which point attempts at perceiving duration are withheld or sabotaged. Similar to these maximally filled durations, minimally filled durations can lead to a perception of temporal stasis (Kramer, 1988, 330).

[25] This experiment is based upon Kramer's informal experiments on the effect of information processing upon perceived duration (Kramer, 1988, 337). Kramer found that musical examples that were 'denser' but of comparable clock-time duration were experienced as being on average $15 \%$ longer than less dense examples.

[26] That is, this is the only example in which less than $50 \%$ of subjects underestimated the duration. In actuality $17 \%$ of subjects perceived the correct actual timing.

[27] Epstein suggested that in works involving multiple tempos, tempos are most often (and most perfectly) related by low order ratios, i.e., 2:1, 1:2, 3:2. Epstein also suggested that complex ratios would (crossculturally) convey an "unsuccessful performance" $(1985,2)$ and that simple proportional ratios are biologically based and therefore "sets ground limits to our aesthetic perceptions" (ibid).

[28] For a different perspective on the question of proportional tempos and tempo relations in Balinese music, see McGraw (in press).

[29] In Finale 2007, the exact shape of tempo changes can be plotted by drawing a line through a temporal grid.

[30] See McGraw (in press), for more detailed tempo maps. Because quality of curve is the focus here tempo and timings are absent. Each of these graphs are based upon expert performances recorded in Bali. In the charts provided above the onset timing of kajar (time keeping gong-chime) strokes is mapped along an $\mathrm{x} / \mathrm{y}$ axis representing tempo and time, respectively (the faster the tempo, the higher its position along the $\mathrm{y}$ axis). Gong strokes are pictured in Example 1 and 7 as larger maroon squares. In the analysis of live recordings a contact microphone was physically placed on the kajar and the timing of the resulting attack points was determined in sound editing software (Audacity) then graphed in an excel spreadsheet.

[31] Anecdotally, several Javanese and American performers of Central Javanese gamelan report feeling that the distance between the second and fourth note of a musical phrase, the gatra, consistently feels shorter, especially in slow tempos, than the distance between the fourth (seleh, weighted goal tone) and the subsequent second (midpoint) tone, although they understood intellectually that objective durations were the same.

\section{REFERENCES}

Amatzia, B. (2001). Musical time organization and space concept: A model of cross-cultural analogy. Ethnomusicology, 45(3), 423-442. 
Amatzia, B. (2006). A cross-cultural structural analogy between pitch and time organizations. Music Perception, 24(3), 265-280.

Becker, J. (1981). Hindu-Buddhist time in Javanese gamelan music. In J.T. Fraser (Ed.), The Study of Time, 4 (pp. 161-172). New York: Springer-Verlag.

Becker, A., \& Becker, J. (1981). A musical icon: Power and meaning in Javanese gamelan music. In W. Steiner (Ed.), The sign in music and literature (203-215). Austin: University of Texas Press.

Block, R.A. \& Zakay D. (1997). Prospective and retrospective duration judgments: A meta-analytical review. Psychonomic Bulletin \& Review, 2, 184-197.

Christensen, T. (1993). Rameau and musical thought in the Enlightenment. Cambridge: Cambridge University Press.

Clarke, E.F. (2001). Meaning and the specification of motion in music. Musicae Scientiae, 5, $213-234$.

Clarke, E.F, \& Krumhansl. C. (1990). Perceiving musical time. Music Perception, 7(3), $213-252$.

Clayton, M. (2000). Time in Indian music: Rhythm, metre, and form in north Indian rag performance. Oxford: Oxford University Press.

Clynes, M., \& and Walker J. (1986). Music as time’s measure. Music Perception, 4(1), 85-119.

Epstein, D. (1985). Tempo relations: A cross-cultural study. Music Theory Spectrum, 7, 34-71.

Epstein, D. (1995). Shaping time: Music, the brain, and performance. New York: Schirmer.

Feld, Steven. (1981). 'Flow like a waterfall': The metaphors of Kaluli musical theory. Yearbook for Traditional Music, 13, 22-47.

Feldman, J., Epstein D., \& Richards W. (1992). Force dynamics of tempo change in music. Music Perception, 10(2), 185-204.

Fraisse, P. (1963). The psychology of time. (J. Leith, Trans.). New York: Harper and Row.

Gibson, J. (1975). Events are perceivable but time is not. In J.T. Fraser (Ed.), The study of time, 2 (pp. 295301). New York: Springer-Verlag.

Greenwald, A.G., Lane K., Banaji M., \& Nosek, B. (2007). Understanding and using the implicit association test: IV, what we know (so far) about the method. In B. Wittenbrink (Ed.), Implicit measures of attitudes (pp. 59-95). New York: Guilford Press.

Johnson, M. (1987). The body in the mind: The bodily basis of meaning, imagination, and reason. Chicago: University of Chicago Press.

Johnson, M., \& Larson S. (2003). 'Something in the way she moves': Metaphors of musical motion. In Gjerdingen (Ed.), Metaphor and Symbol, 18, Proceedings of the $8^{\text {th }}$ International Conference on Music Perception and Cognition, Evanston, Illinois, pp. 63-84.

Keil, C. (1995). The theory of participatory discrepancies: A progress report. Ethnomusicology, 39(1), 1-19.

Kramer, J. D. (1988). The time of music: New meanings, new temporalities, new listening strategies. New York: Schirmer.

Kristofferson, A. B. (1980). A quantal step function in duration discrimination. Perception and Psychophysics, 27, 300-306. 
London, J. (2002). Cognitive constraints on metric systems: some observations and hypotheses. Music Perception, 19 (4), 529-550.

Maceda, J. (1986). A concept of time in a music of Southeast Asia (a preliminary account). Ethnomusicology, 30(1), 11-53.

Mayr, A. (1985). Sketches for a low-frequency solfege. Music Theory Spectrum, 7, 107-113.

McGraw, A. (in press). Different temporalities: The time of Balinese gamelan. Yearbook for Traditional Music, 41.

McPhee, C. (1966). Music in Bali: A study in form and instrumental organization in Balinese orchestral music. New Haven: Yale University Press.

Michon, J.A. (1972). Processing of temporal information and the cognitive theory of time experience. In J.T. Fraser (Ed.), The Study of Time, 1 (pp. 242-257). New York: Springer-Verlag.

Middleton, R. (1990). Studying popular music. Buckingham: Open University Press.

Ornstein, R.E. (1969). On the experience of time. Harmondsworth: Penguin.

Perlman, M. (2004). Unplayed melodies. Berkeley: University of California Press.

Povel, D. \& Essens, P. (1985). Perception of temporal patterns. Music Perception, 2(4), 411-440.

Rammsayer, T. \& Altenmuller E. (2006). Temporal information processing in musicians and nonmusicians. Music Perception, 24(1), 37-48.

Rowell, L. (1979). The subconscious language of musical time. Music Theory Spectrum, 1, 96-106.

Rowell, L. (1985). The temporal 'spectrum'. Music Theory Spectrum, 7, 1-6.

Rowell, L. (1988). Form in the ritual theater music of ancient India. Musica Asiatica, 5, 140-190.

Rudman, L.A., \& Lee, M.R. (2002). Implicit and explicit consequences of exposure to violent and misogynous rap music. Group Processes and Intergroup Relations, 5, 133-150.

Sachs, C. (1953). Rhythm and tempo. New York: W. W. Norton.

Seebass, T. (1996). Change in Balinese musical life: Kebiar in the 1920s and 1930s. In A. Vickers (Ed.), Being modern in Bali: Image and change (pp. 71-91). New Haven: Yale University Southeast Asia Studies.

Seeger, A. (1980). Sing for your sister: The structure and performance of Suya Akia. In N. Mcleod (Ed.), The ethnography of musical performance (pp. 7-43). Norwood, PA: Norwood Editions.

Sundberg, J., \& Verillo V. (1980). On the anatomy of a ritard: A study of timing in music. Journal of the Acoustical Society of America, 68(3), 772-779.

Tenzer, M. (2000). Gamelan Gong Kebyar: The art of twentieth-century Balinese music. Chicago: University of Chicago Press.

Treisman A.M. (1964). Verbal cues, language, and meaning in selective attention. American Journal of Psychology, 77, 206-219.

Walser, R. (1991). The body in the music: Epistemology and musical semiotics. College Music Symposium, 31, 117-136. 
Zagorski-Thomas, S. (2007). The study of groove. Ethnomusicology Forum, 16(2), 327-335.

Zemp, H. (1979). Aspects of 'are' are musical theory. Ethnomusicology, 23, 37-67.

Zuckerkandl, V. (1956). Sound and symbol: Music and the external world. (W.R. Trask, Trans.). London. 